\title{
The Homotopy Analysis Method for a Fourth-Order Initial Value Problems
}

\section{Massoun $\mathbf{Y}^{1 *}$ and Benzine $\mathbf{R}^{2}$}

${ }^{1}$ Department of Mathematics, University of Algiers 1 - Benyoucef Benkhedda, Algeria

${ }^{2}$ Department of Mathematics, University of Annaba, Algeria

\begin{abstract}
In this paper, we apply the homotopy analysis method for solving the fourth-order initial value problems by reformulating them as an equivalent system of first-order differential equations. The analytical results of the differential equations have been obtained in terms of convergent series with easily computable components. Several examples are given to illustrate the efficiency and implementation of the homotopy analysis method.
\end{abstract}

Keywords: Fourth-order initial value problem; Homotopy analysis method

\section{Introduction}

In this section, we consider the general fourth-order initial value problems of the type:

$$
\left\{\begin{array}{rr}
y^{(v i)}(x)=f\left(x, y, y^{\prime}, y^{\prime \prime}, y^{\prime \prime \prime}\right), & a \leq x \leq b, \\
y^{(i)}(a)=\alpha_{i} & i=0, \ldots, 3 .
\end{array}\right.
$$

Where $f$ is continuous function on $[a, b]$ and for each $\mathrm{i}=0,3 \ldots$ the parameters $\alpha_{\mathrm{i}}$ are real constants. Such type of initial value problems arise in the mathematical modeling of physical and engineering sciences. Liao [1-5] presented the homotopy analysis method for solving different problems in several dimensions. In this method, produced solution is in infinite series form and this form is converges to the exact solution.

In this paper, we applies the homotopy analysis method to solve the linear and nonlinear fourth-order initial value problems by using the transformation of problem to a system of first-order differential eqn. (1). Several Numerical examples have been presented in Section 3, to illustrate the advantage and the effectiveness of the proposed method.

\section{Homotopy Analysis Method}

In this section, we will briefly describe the use of HAM for the differential equation:

$$
N[u(x)]=g(x)
$$

Where $N$ is a linear or nonlinear operators, $x$ denote the independent variable, $u(x)$ is an unknown function and $g(x)$ is a known analytic function.

By means of generalizing the traditional homotopy analysis method, Liao [1] constructs the so-called zeroth-order deformatin equation:

$$
(1-q) L\left[\phi(x, q)-u_{0}(x)\right]=c q H(x)\{N[\phi(x, q)-g(x)]\}
$$

Where $L$ is an auxiliary linear operator with the property $L[0]=0 \mathrm{~N}$ is the nonlinear operator related to the original eqn. $(2), \mathrm{q} \in[0,1]$ is the embedding parameter in topology (called the homotopy parameter), $c^{1} 0$ is the so called "convergence-control parameter", and $H(x)$ is an auxiliary function [6-9].

Note that in the frame of the homotopy, we have great freedom to choose the auxiliary linear operator $L$, the initial guess $u_{0}(x)$ the auxiliary function $H(x)$ and the convergence-control-parameter $c$.

Obviously, when $q=0$ and $q=1$ one has:

$$
\phi(x, 0)=u_{0}(x) \text { and } \phi(x, 1)=u(x)
$$

thus as $q$ increases from 0 to 1 , the solution $\varphi(x, q)$ varies from the initial guesses $u_{0}(x)$ to the exact solution $u(x)$

Expanding $\varphi_{i}(x, q)$ in Taylor series with respect to $q$ one has:

$$
\phi(x, q)=u_{0}(x)+\sum_{m=1}^{+\infty} u_{m}(x) q^{m}
$$

where:

$$
u_{m}(x)=\left.\frac{1}{m !} \frac{\partial^{m} \phi(x, q)}{\partial q^{m}}\right|_{q=0}
$$

if the auxiliary linear operator, the initial guesses, the auxiliary parameters $c$, and the auxiliary function are so properly chosen, the series solutions eqn. (4) converge at $q=1$, and we have:

$$
\phi(x, 1)=u_{0}(x)+\sum_{i=1}^{+\infty} u_{m}(x)
$$

wich must be of the solution of the original nonlinear equations, as proved by Liao [1].

$$
\begin{aligned}
& \text { As c=-1 eqn. (2) becomes: } \\
& (1-q) L\left[\phi(x, q)-u_{0}(x)\right]-q\{N[\phi(x, q)-g(x)]=0
\end{aligned}
$$

which is used mostly in the homotopy perturbation method (HPM) [2].

According to eqn. (5), the governing equations can be deduced from the zeroth-order deformation eqn. (2). Define the vector:

$$
\vec{u}_{n}(x)=\left\{u_{0}(x), u_{1}(x), \ldots, u_{n}(x),\right\}
$$

Differentiating eqn. (2) $m$ times with respect to the embedding parameter $\mathrm{q}$ and then setting $q=0$ and finally dividing them by, $m$ ! we have the so-called mth-order deformation equation:

$$
L\left[u_{m}(x)-\chi_{m} u_{m-1}(x)\right]=c H(x) R_{m}\left(\vec{u}_{m-1}\right)
$$

*Corresponding author: Massoun Y, Professor, Department of Mathematics University of Algiers 1 - Benyoucef Benkhedda, Algeria, Tel: 256485445; E-mail youcef.ens@hotmail.fr

Received January 15, 2018; Accepted March 16, 2018; Published March 20 2018

Citation: Massoun Y, Benzine R (2018) The Homotopy Analysis Method for a Fourth-Order Initial Value Problems. J Phys Math 9: 265. doi: 10.4172/20900902.1000265

Copyright: (c) 2018 Massoun Y, et al. This is an open-access article distributed under the terms of the Creative Commons Attribution License, which permits unrestricted use, distribution, and reproduction in any medium, provided the original author and source are credited. 
where

$$
\begin{aligned}
& R_{m}\left(\vec{u}_{m-1}\right)=\left.\frac{1}{(m-1) !} \frac{\partial^{m-1} N[\phi(x, q)]}{\partial q^{m-1}}\right|_{q=0} \\
& \text { and }
\end{aligned}
$$

$$
\chi_{m}= \begin{cases}0, & m \leq 1 \\ 1, & m>1\end{cases}
$$

It should be emphasized that $u_{m}(x), m \geq 1$ is governed by the linear eqn. (6) with the linear initial conditions that come from the original problem, which can be easily solved by symbolic computation softwares such as Maple and Mathematica [10].

\section{The modified homotopy analysis method}

In this part, we will briefly describe use of the HAM for problem eqn. (1) the modified HAM suggests that we reformulating the problem eqn. (1) as an equivalent system of a first-order differential equation, we use the transformation:

$$
y_{1}(x)=y(x), \quad \frac{d y}{d x}=y_{2}(x), \quad \frac{d^{2} y}{d x^{2}}=y_{3}(x), \quad \frac{d^{3} y}{d x^{3}}=y_{4}(x),
$$

we can rewrite the fourth-order initial value problem eqn. (1) as the system of ordinary differential equations:

$$
\left\{\begin{array}{lcc}
y_{1}^{\prime}(x)= & y_{2}(x) \\
y_{2}^{\prime}(x)= & y_{3}(x) \\
y_{3}^{\prime}(x)= & y_{4}(x) \\
y_{4}^{\prime}(x)= & f\left(x, y_{1}(x), y_{2}(x), y_{3}(x), y_{4}(x)\right)
\end{array}\right.
$$

with the initial conditions:

$$
\left\{\begin{array}{l}
y_{1}(a)=y(a) \\
y_{2}(a)=y^{\prime}(a) \\
y_{3}(a)=y^{\prime \prime}(a) \\
y_{4}(a)=y^{\prime \prime \prime}(a)
\end{array}\right.
$$

We will present a brief description of the standard HAM for a system of ODEs, we consider the differential equation:

$$
N_{i}\left[u_{i}(x)\right]=g_{i}(x)
$$

where $N_{i}$ are nonlinear operators (and i-1..4) and $x$ denote the independent variable, $\mathrm{u}_{\mathrm{i}}(x)$ are unknown functions and gi( $\left.\mathrm{x}\right)$ are known analytic functions, for gi $(\mathrm{x})=0$ eqn. (11) reduces to the homogeneous equation [11-13].

By means of generalizing the traditional homotopy analysis method, Liao constructs the so-called zeroth-order deformatin equation [1]:

$$
(1-q) L\left[\phi_{i}(x, q)-u_{i, 0}(x)\right]=c_{i} q H(x)\left\{N_{i}\left[\phi_{i}(x, q)-g_{i}(x)\right]\right\}
$$

Where $L$ is an auxiliary linear operator with the property $L[0]=0 \mathrm{~N}$ is the nonlinear operator related to the original eqn. (1) $q \in[0,1]$ is the embedding parameter in topology (called the homotopy parameter), $c_{i}^{1} 0$ is the so called "convergence-control parameter", and $H(x)$ is an auxiliary function [14].

Note that in the frame of the homotopy, we have great freedom to choose the auxiliary linear operator $L$, the initial guess $u_{i 0}(x)$, the auxiliary function $H(x)$ and the convergence-control-parameter $c_{i}$.

Obviously, when $q=0$ and $q=1$ one has:

$$
\phi_{i}(x, 0)=u_{i, 0}(x) \text { and } \phi_{i}(x, 1)=u_{i}(x)
$$

thus as q increases from 0 to, 1 the solution $\varphi_{\mathrm{i}}(x, q)$ varies from the initial guesses $u_{i, 0}(x)$ to the solutions $u_{i}(x)$
Expanding $\varphi_{\mathrm{i}}(x, q)$ in Taylor series with respect to $q$ one has:

$$
\phi_{i}(x, q)=u_{i, 0}(x)+\sum_{m=1}^{+\infty} u_{i, m}(x) q^{m}
$$

where:

$$
u_{i, m}(x)=\left.\frac{1}{m !} \frac{\partial^{m} \phi_{i}(x, q)}{\partial q^{m}}\right|_{q=0}
$$

if the auxiliary linear operator, the initial guesses, the auxiliary parameters $c_{i}$ and the auxiliary function are so properly chosen, the series solutions in eqn. (14) converge at $q=1$ and we have:

$$
\phi_{i}(x, 1)=u_{i, 0}(x)+\sum_{i=1}^{+\infty} u_{i, m}(x)
$$

wich must be of the solutions of the original nonlinear equations, as proved by Liao [1].

$$
\begin{aligned}
& \text { As } c_{i}=-12 \text { eqn. (12) and } H(x)=1 \text { becomes: } \\
& (1-q) L\left[\phi_{i}(x, q)-u_{i, 0}(x)\right]-q\left\{N_{i}\left[\phi_{i}(x, q)-g_{i}(x)\right]=0\right.
\end{aligned}
$$

which is used mostly in the homotopy perturbation method (HPM) [2].

According to eqn. (15) the governing equations can be deduced from the zeroth-order deformation eqn. (12).Define the vector:

$$
\vec{u}_{i, n}=\left\{u_{i, 0}(x), u_{i, 1}(x), \ldots, u_{i, n}(x),\right\}
$$

Differentiating eqn. (12) $m$ times with respect to the embedding parameter $q$ and then setting $q=0$ and finally dividing them by $m$ ! we have the so-called $\mathrm{mth}$-order deformation equation:

$$
L\left[u_{i, m}(x)-\chi_{m} u_{i, m-1}(x)\right]=c_{i} H_{i}(x) R_{i, m}\left(\vec{u}_{i, m-1}\right)
$$

where

$$
R_{i, m}\left(\vec{u}_{i, m-1}\right)=\frac{1}{(m-1) !} \frac{\partial^{m-1} N_{i}\left[\phi_{i}(x, q)\right]}{\partial q^{m-1}}
$$

and

$$
\chi_{m}= \begin{cases}0, & m \leq 1 \\ 1, & m>1\end{cases}
$$

It should be emphasized that $u_{m}(t), m \geq 1$ is governed by the linear eqn. (16) with the linear initial conditions that come from the original problem, which can be easily solved by symbolic computation softwares such as Maple and Mathematica.

\section{Application}

In this section, we shall apply HAM to several test examples to demonstrate its accuracy. To show the efficiency of the present method for our problem in comparison with the exact solution we report absolute error which is defined by:

$$
E_{i}=\left|u_{i, \text { exact }}(x)-u_{i, n}(x)\right|, \quad i=1, \ldots, 4
$$

where $u_{i, n}(x)=u_{i, 0}(x)+u_{i, 1}(x)+\ldots+u_{i, n}(x)$

\section{Example 3.1}

Consider the following non linear fourth-order problem:

$$
y^{(4)}(x)=y^{\prime 2}(x)-y(x) y^{(3)}(x)-4 x^{2}+e^{x}\left(1-4 x+x^{2}\right), \quad 0 \leq x \leq 1
$$

with the following boundary conditions:

$$
y(0)=1 \quad y^{\prime}(0)=1 \quad y^{\prime \prime}(0)=3 \quad y^{(3)}(0)=1
$$


Citation: Massoun Y, Benzine R (2018) The Homotopy Analysis Method for a Fourth-Order Initial Value Problems. J Phys Math 9: 265. doi: 10.4172/2090-0902.1000265

Page 3 of 4

The analytic solution is:

$y(x)=x^{2}+e^{x}$

Using the transformation from eqn. (8) we can rewrite the fourthorder initial value problem in eqn. (19) as the system of ordinary differential equations:

$$
\left\{\begin{array}{lcc}
y_{1}^{\prime}(x) & = & y_{2}(x) \\
y_{2}^{\prime}(x) & y_{3}(x) \\
y_{3}^{\prime}(x) & = & y_{4}(x) \\
y_{4}^{\prime}(x)= & y_{2}^{2}(x)-y_{1}(x) y_{3}(x)-4 x^{2}+e^{x}\left(1-4 x+x^{2}\right)
\end{array}\right.
$$

with the initial conditions:

$$
y_{1}(0)=1 \quad y_{2}(0)=1 \quad y_{3}(0)=3 \quad y_{4}(0)=1
$$

To solve eqn. (21) by means of the standard HAM, we choose the initial approximations:

$$
y_{1,0}(0)=1 \quad y_{2,0}(0)=1 \quad y_{3,0}(0)=3 \quad y_{4,0}(0)=1
$$

and the linear operator:

$$
L\left[\phi_{i}(x, q)\right]=\frac{\partial \phi_{i}(x, q)}{\partial x}, \quad i=1, \ldots, 4
$$

with the property:

$$
L\left[c_{i}\right]=0, \quad i=1, \ldots, 4
$$

Where $c_{i}$ are constants of integrations. Furthermore, eqn. (21) suggest that we define a system of linear operators as:

$$
\left\{\begin{array}{c}
N_{i}\left[\phi_{i}(x, q)\right]=\frac{\partial \phi_{i}(x, q)}{\partial x}-\phi_{i+1}(x), \quad i=1, \ldots, 3 \\
N_{4}\left[\phi_{4}(x, q)\right]=\phi_{4}(x, q)-\left(\phi_{1}\right)^{2}(x, q)+\phi(x, q) \phi^{(3)}(x, q) \\
+4 x^{2}-e^{x}\left(x^{2}-4 x+1\right)
\end{array}\right.
$$

According to eqn. (12), we construct the zeroth-order deformation equation:

$$
(1-q) L\left[\phi_{i}(x, q)-u_{i, 0}(x)\right]=c_{i} q H(x)\left\{N_{i}\left[\phi_{i}(x, q)-g_{i}(x)\right]\right\}
$$

and the mth-order deformation equation (for $H_{i}(x)=1$ ),

$$
L\left[u_{i, m}(x)-\chi_{m} u_{i, m-1}(x)\right]=c_{i} R_{i, m}\left(\vec{u}_{i, m-1}\right)
$$

with the initial conditions:

$$
u_{1,0}(0)=1 \quad u_{2,0}(0)=1 \quad u_{3,0}(0)=3 \quad u_{4,0}(0)=1
$$

where

$$
\left\{\begin{aligned}
R_{i, m}\left(\vec{u}_{i, m-1}\right)= & u_{i, m-1}^{\prime}(x)-u_{i+1, m-1}(x), \quad i=1, \ldots, 3 \\
R_{4, m}\left(\vec{u}_{4, m-1}\right)= & u_{4, m-1}^{\prime}-u_{1, m-1}^{2}(x)+u_{1, m-1}(x) u_{4, m-1}(x) \\
& +4 x^{2}-e^{x}\left(x^{2}-4 x+1\right)
\end{aligned}\right.
$$

Now, the solution of the mth-order deformation eqn (22) for $m \geq 1$ becomes:

$$
u_{i, m}(x)=\chi_{m} u_{i, m-1}(x)+c_{i} \int_{0}^{1} R_{i, m}\left(\vec{u}_{i, m-1}\right) d x+\alpha_{i}, \quad i=1, \ldots, 4
$$

where the constants of integrations $\alpha_{i},(i=1, \ldots, 4)$ are determined by the initial conditions:

Then the series solutions expression by the standard HAM can be written for $i=1,2,3,4$ in the form:

$$
u_{i}(x)=u_{i, 0}(x)+u_{i, 1}(x)+u_{i, 2}(x)+\ldots
$$

Table 1 exhibits the exact solution and the series solution along with the errors obtained by using the homotopy analysis method [1315]. It is obvious that the errors can be reduced further and higher accuracy can be obtained by evaluating more components of $u(x)$.

\section{Example 3.2}

Consider the following nonlinear initial fourth-order problem:

$$
\left\{\begin{array}{c}
y^{(4)}(x)-4 y^{\prime \prime}(x)=0, \quad 0 \leq x \leq 1 \\
y(0)=1, \quad y^{\prime}(0)=3, \quad y^{\prime \prime}(0)=0, \quad y^{\prime \prime \prime}(0)=16
\end{array}\right.
$$

The analytic solution is:

$$
y(x)=1-x+e^{2 x}-e^{-2 x}
$$

Using the transformation eqn. (8) we can rewrite the fourth-order initial value problem as the system of ordinary differential equations:

$$
\left\{\begin{array}{l}
y_{1}^{\prime}(x)=y_{2}(x) \\
y_{2}^{\prime}(x)=y_{3}(x) \\
y_{3}^{\prime}(x)=y_{4}(x) \\
y_{4}^{\prime}(x)=4 y_{3}(x)
\end{array}\right.
$$

with the initial conditions:

$$
y_{1}(0)=1, \quad y_{2}(0)=3, \quad y_{3}(0)=0, \quad y_{4}(0)=16 .
$$

as shown in Table 2 the series solutions ( $10^{\text {th }}$ order) to get the analytic solution $y(t)=1-x+e^{2 x}-e^{-2 x}$ which are coinciding with the exact solution.

\section{Example 3.3}

We consider the nonlinear fourth-order initial problem:

\begin{tabular}{|l|l|l|l|}
\hline $\mathbf{x}$ & Exact solution & Series solution & Error $(\mathbf{a})$ \\
\hline 0 & 1 & 1 & $0 . \mathrm{E}-96$ \\
\hline 0.1 & 1.115170918 & 1.115155544 & $1.53739 \mathrm{E}-5$ \\
\hline 0.2 & 1.261402758 & 1.261378273 & $2.44842 \mathrm{E}-5$ \\
\hline 0.3 & 1.439858807 & 1.439812900 & $4.59070 \mathrm{E}-5$ \\
\hline 0.4 & 1.651824697 & 1.651719012 & $1.05685 \mathrm{E}-5$ \\
\hline 0.5 & 1.898721270 & 1.898488975 & $2.32295 \mathrm{E}-4$ \\
\hline 0.6 & 1.182118800 & 2.181669975 & $4.49236 \mathrm{E}-4$ \\
\hline 0.7 & 2.503752707 & 2.502987975 & $7.64732 \mathrm{E}-4$ \\
\hline 0.8 & 2.865540928 & 2.864382992 & $1.15794 \mathrm{E}-3$ \\
\hline 0.9 & 3.269603111 & 3.268042230 & $1.56088 \mathrm{E}-3$ \\
\hline 1 & $1+\mathrm{e}$ & 3.176446454 & $1.83537 \mathrm{E}-3$ \\
\hline
\end{tabular}

Table 1: List of exact solution - series solution.

\begin{tabular}{|l|l|l|l|}
\hline $\mathbf{x}$ & Exact solution & Series solution & Error $(\mathbf{a})$ \\
\hline 0 & 1 & 1 & $0 . \mathrm{E}-99$ \\
\hline 0.1 & 1.302672005 & 1.302672005 & $2.59792 \mathrm{E}-14$ \\
\hline 0.2 & 1.621504651 & 1.621504651 & $9.20886 \mathrm{E}-12$ \\
\hline 0.3 & .973307164 & .973307163 & $4.20469 \mathrm{E}-10$ \\
\hline 0.4 & 2.376211964 & 2.376211957 & $7.33788 \mathrm{E}-9$ \\
\hline 0.5 & 2.850402387 & 2.850402314 & $7.23649 \mathrm{E}-8$ \\
\hline 0.6 & 3.418922710 & 3.418922223 & $4.87444 \mathrm{E}-7$ \\
\hline 0.7 & 4.108603002 & 4.108600504 & $2.49845 \mathrm{E}-6$ \\
\hline 0.8 & 4.951135906 & 4.951125477 & $1.04290 \mathrm{E}-5$ \\
\hline 0.9 & 5.984348576 & 5.984311473 & $3.71028 \mathrm{E}-5$ \\
\hline 1 & 7.253720815 & 7.253604649 & $1.16166 \mathrm{E}-4$ \\
\hline
\end{tabular}

Table 2: Variation of exact solution - series solution with $y(t)=\sin x$. 


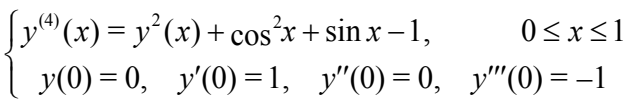

The exact solution is:

$$
y(x)=\sin (x)
$$

Using the transformation, we can rewrite the fourth-order initial value problem as the system of ordinary differential equations:

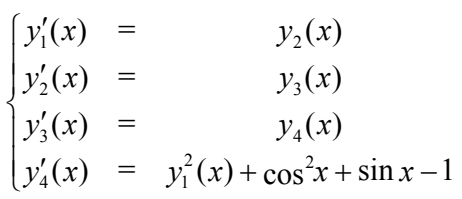

with the initial conditions:

$$
y_{1}(0)=0, \quad y_{2}(0)=1, \quad y_{3}(0)=0, \quad y_{4}(0)=-1 .
$$

as shown in Table 3 the series solutions ( $10^{\text {th }}$ order) to get the exact solution $\mathrm{y}(\mathrm{x})=\sin (\mathrm{x})$ which are coinciding with the exact solution.

\section{Example 3.4}

Consider the linear system of fourth-order initial value problem:

$$
\left\{\begin{array}{ccccc}
y^{(4)}(x)=e^{3 x} u(x), & y(0)=1 & y^{\prime}(0)=-1 & y^{\prime \prime}(0)=1 & y^{(3)}(0)=-1 \\
z^{(4)}(x)=16 e^{-x} y(x), & z(0)=1 & z^{\prime}(0)=-2 & z^{\prime \prime}(0)=4 & z^{(3)}(0)=-8 \\
w^{(4)}(x)=81 e^{-x} z(x), & w(0)=1 & w^{\prime}(0)=-3 & w^{\prime \prime}(0)=9 & w^{(3)}(0)=-27 \\
u^{(4)}(x)=256 e^{-x} w(x), & u(0)=1 & u^{\prime}(0)=-4 & u^{\prime \prime}(0)=16 & u^{(3)}(0)=-64
\end{array}\right.
$$

The analytic solution is:

$$
y(x)=e^{-x} \quad z(x)=e^{-2 x} \quad w(x)=e^{-3 x} \quad u(x)=e^{-4 x}
$$

Using the transformation we can rewrite the system of ODEs from eqn. (23)as the system of ordinary differential equations:

$$
\left\{\begin{array}{cccc}
y_{1}(x)=y(x) & z_{1}(x)=z(x) & w_{1}(x)=w(x) & u_{1}(x)=u(x) \\
y_{2}(x)=y^{\prime}(x) & z_{2}(x)=z^{\prime}(x) & w_{2}(x)=w^{\prime}(x) & u_{2}(x)=u^{\prime}(x) \\
y_{3}(x)=y^{\prime \prime}(x) & z_{3}(x)=z^{\prime \prime}(x) & w_{3}(x)=w^{\prime \prime}(x) & u_{3}(x)=u^{\prime \prime}(x) \\
y_{4}(x)=y^{(3)}(x) & z_{4}(x)=z^{(3)}(x) & w_{4}(x)=w^{(3)}(x) & u_{4}(x)=u^{(3)}(x)
\end{array}\right.
$$

with the initial conditions:

$$
\left\{\begin{array}{cccc}
y_{1}(0)=1 & z_{1}(0)=1 & w_{1}(0)=1 & u_{1}(0)=1 \\
y_{2}(0)=-1 & z_{2}(0)=-2 & w_{2}(0)=-3 & u_{2}(0)=-4 \\
y_{3}(0)=1 & z_{3}(0)=4 & w_{3}(0)=9 & u_{3}(0)=16 \\
y_{4}(0)=-1 & z_{4}(0)=-8 & w_{4}(0)=-27 & u_{4}(0)=-64
\end{array}\right.
$$

as shown in Table 4 exhibits the errors obtained by using the approximate solution of the homotopy analysis method.

\begin{tabular}{|l|l|l|l|}
\hline $\mathbf{X}$ & Exact solution & Series solution & Error (a) \\
\hline 0 & 1 & $0 . \mathrm{E}-101$ & $0 . \mathrm{E}-99$ \\
\hline 0.1 & 0.099833416 & 0.099831873 & $1.54285 \mathrm{E}-6$ \\
\hline 0.2 & 0.198669330 & 0.198666394 & $2.93657 \mathrm{E}-6$ \\
\hline 0.3 & 0.295520206 & 0.295516161 & $4.04534 \mathrm{E}-6$ \\
\hline 0.4 & 0.389418342 & 0.389413600 & $4.74179 \mathrm{E}-6$ \\
\hline 0.5 & 0.479425538 & 0.479420671 & $4.86696 \mathrm{E}-6$ \\
\hline 0.6 & 0.564642473 & 0.564638339 & $4.13389 \mathrm{E}-6$ \\
\hline 0.7 & 0.644217687 & 0.644215735 & $1.95201 \mathrm{E}-6$ \\
\hline 0.8 & 0.717356090 & 0.717358935 & $2.84436 \mathrm{E}-6$ \\
\hline 0.9 & 0.783326909 & 0.783339275 & $1.23663 \mathrm{E}-5$ \\
\hline 1 & $\sin 1$ & 0.844501103 & $3.01182 \mathrm{E}-5$ \\
\hline
\end{tabular}

Table 3: Variation of exact solution - series solution with Error (a).

\begin{tabular}{|l|l|l|l|l|}
\hline $\mathbf{x}$ & Error of $\mathbf{y}(\mathbf{x})$ & Error of $\mathbf{z}(\mathbf{x})$ & Error of $\mathbf{w}(\mathbf{x})$ & Error of $\mathbf{u}(\mathbf{x})$ \\
\hline 0 & $0 . \mathrm{E}-93$ & $0 . \mathrm{E}-99$ & $0 . \mathrm{E}-97$ & $0 . \mathrm{E}-96$ \\
\hline 0.1 & $7.54521 \mathrm{E}-7$ & $1.70883 \mathrm{E}-9$ & $5.07700 \mathrm{E}-7$ & $4.02179 \mathrm{E}-6$ \\
\hline 0.2 & $1.12924 \mathrm{E}-5$ & $1.64622 \mathrm{E}-8$ & $4.16492 \mathrm{E}-6$ & $2.32361 \mathrm{E}-5$ \\
\hline 0.3 & $5.51765 \mathrm{E}-5$ & $3.16353 \mathrm{E}-8$ & $1.34459 \mathrm{E}-5$ & $6.74763 \mathrm{E}-5$ \\
\hline 0.4 & $1.68971 \mathrm{E}-4$ & $1.26221 \mathrm{E}-7$ & $2.85145 \mathrm{E}-5$ & $1.62335 \mathrm{E}-4$ \\
\hline 0.5 & $3.99793 \mathrm{E}-4$ & $9.49274 \mathrm{E}-7$ & $4.78585 \mathrm{E}-5$ & $3.63740 \mathrm{E}-4$ \\
\hline 0.6 & $8.01764 \mathrm{E}-4$ & $3.55305 \mathrm{E}-6$ & $6.78252 \mathrm{E}-5$ & $7.77619 \mathrm{E}-4$ \\
\hline 0.7 & $1.43001 \mathrm{E}-3$ & $1.01404 \mathrm{E}-5$ & $8.14644 \mathrm{E}-5$ & $1.58613 \mathrm{E}-3$ \\
\hline 0.8 & $2.33066 \mathrm{E}-3$ & $2.46804 \mathrm{E}-5$ & $7.64867 \mathrm{E}-5$ & $3.07537 \mathrm{E}-3$ \\
\hline 0.9 & $3.52584 \mathrm{E}-3$ & $5.38602 \mathrm{E}-5$ & $3.19459 \mathrm{E}-5$ & $5.65814 \mathrm{E}-3$ \\
\hline 1 & $4.99021 \mathrm{E}-3$ & $1.08370 \mathrm{E}-4$ & $8.67341 \mathrm{E}-5$ & $9.88643 \mathrm{E}-3$ \\
\hline
\end{tabular}

Table 4: Error is related with exact solution and series solution.

\section{Conclusion}

In this paper, the homotopy analysis method HAM has been successfully used for finding the solution of special forth-order initial value problem under the the transformation of the fourth-order value problem to the system of the first order differential equation. Several examples showed the validity and potential of this modification of the homotopy analysis method for linear and nonlinear special fourthorder initial value problem in the science and engineering.

\section{References}

1. Liao SJ (2012) Homotopy Analysis Method in Non Linear Differential Equations Springer, New York.

2. LiaoSJ (2003) Beyond Perturbation - Introduction to the Homotopy Analysis Method and its applications. Chapman and Hall/ CRC Press.

3. Liao SJ (1992) The Proposed Homotopy Analysis Technique for the Solution of Nonlinear Problems, PhD thesis, Shanghai Jiao Tong University, Shanghai.

4. Liao SJ (2005) Comparison between the homotopy analysis method and homotopy perturbation method. Appl Math Comput 169: 118-694.

5. Liao SJ (2004) On the homotopy analysis method for nonlinear problems. App Math Comput 147: 499513

6. Awoyemi DO (2007) Algorithmic collocation approach for direct solution of fourth-order initial value problems of ordinary differential equations. International Journal of Computer Mathematics 82: 321-329.

7. Hussaina K, Ismaila F, Senu N (2016) Solving Directly Special Fourth-Orde Ordinary Differential Equations Using Runge-Kutta Type Method. Journal of Computational and Applied Mathematics 306: 179-199.

8. El-Gamel M, Behiry SH, Hashish H (2003) Numerical method for the solution of special nonlinear fourth-order boundary value problems. Applied Mathematics and Computation 145: 717-734.

9. Al-Khaled K, Anwar MN (2007)Numerical comparison of methods for solving second-order ordinary initial value problems, Applied Mathematical Modelling 31: 292-301.

10. Krishna CBR, Rao PSRC (2014) Study of Stability Analysis for a Class of Fourth Order Boundary Value Problems. Applied Mathematics 5: 1887-1893.

11. Golbabai A, Javidi M (2007) Application of homotopy perturbation method for solving eight-order boundary value problems. Applied Mathematics and Computation 8: 334-346.

12. NoorMA, Mohyud-Din ST (2008) Homotopy perturbation method for solving sixth-order boundary value problems. Computers and Mathematics with Applications 55: 2953-2972.

13. Momani S, Noor MA (2007) Numerical comparison of methods for solving a special fourth-order boundary value problem. Applied Mathematics and Computation 191: 218-224.

14. Boutayeb A, Twizell EH (1992) Numerical methods for the solution of special sixth-order boundary value problems. Int J Comput Math 45: 207-233

15. Bataineh AS, Noorani MSM, Hashim I, Bataineh AS, Noorani MSM, et al. (2008) Solving systems of ODEs by homotopy analysis method. Commun Nonlin Sci Numer Simul 13: 2060-2070. 